\title{
DOSAGE DU SACCHAROSE ET DU LACTOSE DANS LE LAIT CONDENSÉ SUCRÉ (1)
}

\author{
par \\ J. BONNET \\ Chargé de cours. Chaire de Technologie.
}

Au cours de ces deux dernières années, nous avons eu l'occasion, à de nombreuses reprises, d'utiliser la méthode préconisée par F. Richand (Le Lait, 1927, p. 635) pour la détermination du saccharose et du lactose dans le lait condensé sucré.

Le principe est le suivant : déterminer l'action réductrice du lait condensé sur la liqueur de Fehling avant et après inversion.

On opère de la façon suivante :

$1^{\circ}$ Peser 50 grammes de lait condensé dans un ballon stift de $100 \mathrm{em}^{3}$; dissoudre dans l'eau distillée et porter à 100. C'est la solution A.

$2^{\circ}$ Introduire $10 \mathrm{~cm}^{3}$ de la solution A dans un ballon de $100 \mathrm{~cm}^{3}$; diluer, puis ajouter quelques gouttes d'acide acétique concentré ; porter au volume, agiter et laisser reposer ; lorsque le coagulum est bien formé, filtrer. C'est la solution M dont $100 \mathrm{~cm}^{3}$ contiennent 5 grammes de lait condensé.

$3^{\circ}$ Déterminer l'action réductrice de cette solution M sur la liqueur de Fehling normale $\left(1 \mathrm{~cm}^{3}\right.$ correspond à 5 milligrammes de glucose). Soit $\mathrm{x} \mathrm{cm}^{3}$ de solution pour réduire $10 \mathrm{~cm}^{3}$ de liqueur de Fehling. La quantité de glucose équivalente pour 100 grammes de lait condensé est égale à $\frac{100}{\mathrm{x}}$.

$4^{\circ}$ Prélever $25 \mathrm{~cm}^{3}$ de solution $M$ dans un vase de Berlin (nous préférons prélever $25 \mathrm{~cm}^{3}$, au lieu de 50 préconisés par l'auteur, de façon à utiliser un nombre de centimètres cubes de solution voisine de celui de la liqueur de Fehling; il est, en effet, toujours recommandé d'avoir dess volumes de solutions réductrices et de liqueur de Fehling à peu près équivalents).

A ces $25 \mathrm{~cm}^{3}$ de solution $\mathrm{M}$, ajouter 5-6 gouttes de $\mathrm{HCl}$ concentré ; faire bouillir 10 minutes; neutraliser au carbonate de soude, refroidir, puis verser dans un ballon de $100 \mathrm{~cm}^{3}$ et porter au volume. C'est la solution. $\mathrm{N}$, dont $100 \mathrm{~cm}^{3}$ contiennent $1 \mathrm{gr}$. 25 de lait condensé.

$5^{\circ}$ Déterminer le pouvoir réducteur de cette solution $\mathrm{N}$ comme précédemment sur $10 \mathrm{~cm}^{3}$ de liqueur de Fehling. Soit y $\mathrm{cm}^{3}$ de solution.

(1) Bulletin de l'Institut Agronomique et des Stations de Recherches de Gembloux. t. VIII, nos 3-4, août-novembre 1939. 
La quantité de glucose équivalente à 100 de lait condensé est égale à $\frac{400}{\mathrm{y}}$.

$6^{\circ}$ Le glucose dû au sucre interverti est donc:

$$
\frac{400}{y}-\frac{100}{x}
$$

Le saccharose correspondant $=\left(\frac{400}{y}-\frac{100}{x}\right) \frac{95}{100}$.

$7^{\circ}$ Détermination du lactose.

L'action réductrice de la solution $\mathrm{M}$ avant inversion est due au lactose. Pour obtenir celui-ci, il suffit, d'après l'auteur, de multiplier la quantité de glucose trouvée par le facteur 1,58.

Il nous semble que ce facteur est quelque peu supérieur à la réalité. Au cours de nombreux essais, nous ne sommes jamais parvenu à le reproduire.

Voici les moyennes auxquelles nous sommes arrivés en partant de solutions de lactose pur, pro analysi :

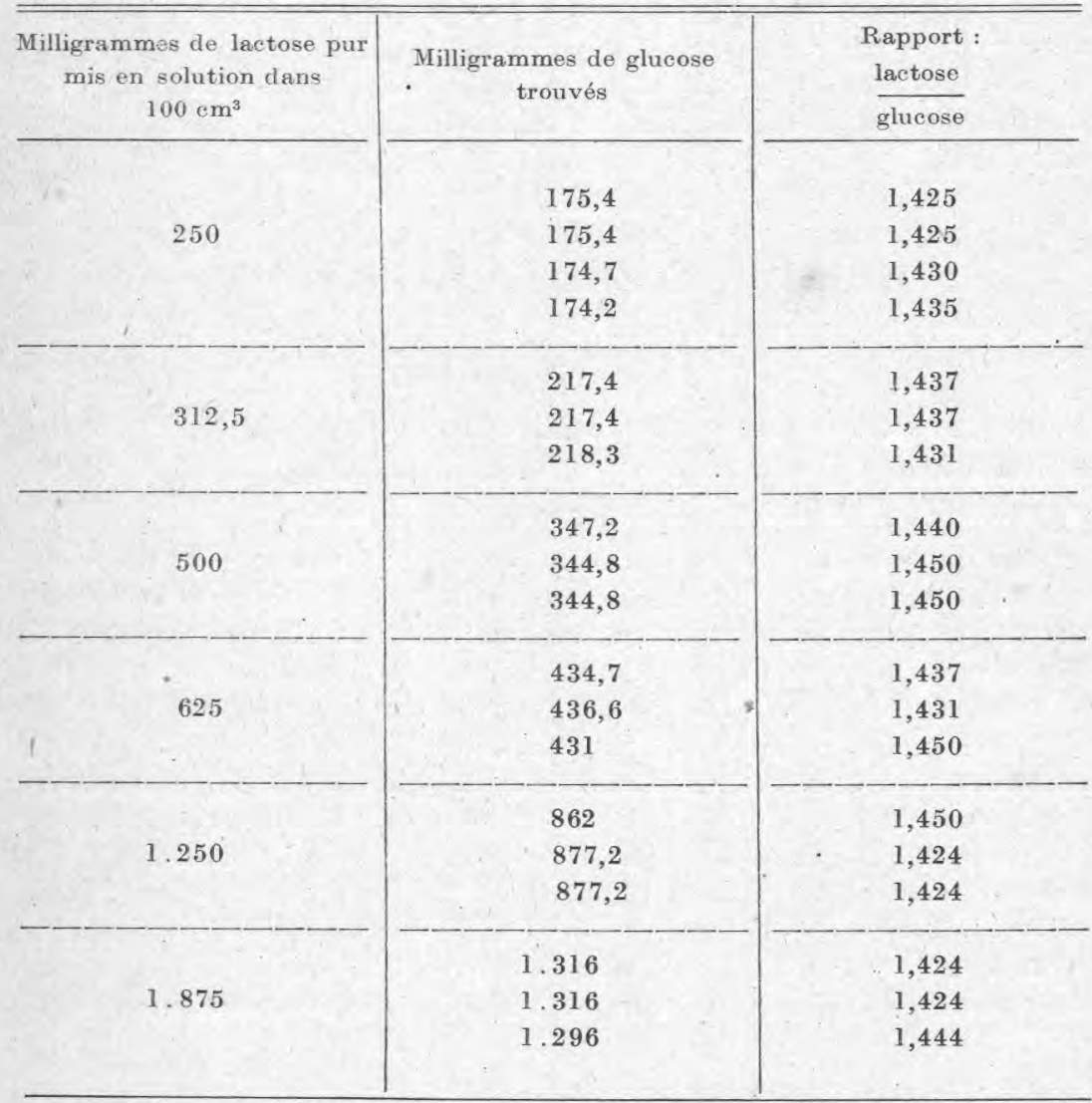


Nous obtiendrons, d'autre part, des chiffres très voisins de ceuxci en opérant par calcul et en utilisant les tables de G. BERTrand donnant les poids de lactose et de glucose en fonction des poids de cuivre précipité :

\begin{tabular}{|c|c|c|c|}
\hline $\begin{array}{l}\text { Poids de cuivre } \\
\text { en milligrammes }\end{array}$ & $\begin{array}{c}\text { Lactose } \\
\text { correspondant } \\
\text { eu milligrammes }\end{array}$ & $\begin{array}{c}\text { Glucose } \\
\text { correspondant } \\
\text { en milligrammes }\end{array}$ & $\begin{array}{c}\text { Rapport : } \\
\frac{\text { lactose }}{\text { glucose }}\end{array}$ \\
\hline 20,4 & 14,29 & 10 & 1,429 \\
\hline 40,1 & 28,5 & 20 & 1,426 \\
\hline 59,3 & 43 & 30 & 1,433 \\
\hline 77,5 & $57^{\circ}$ & 40 & 1,425 \\
\hline 95,4 & 71 & 50 & 1,420 \\
\hline 112,8 & 84,9 & 60 & 1,415 \\
\hline 129,8 & 98,7 & 70 & 1,41 \\
\hline
\end{tabular}

En conséquence, nous présumons que le facteur 1,43 s'approche plus de la réalité.

La quantité de glucose trouvée lors de la réduction de la liqueur de Fehling par la solution M, et multipliée par 1,43, nous donnera done le lactose dans 100 grammes de lait condensé.

\section{BIBLIOGRAPHIE ANALYTIQUE}

\section{LES LIVRES}

\section{Mignolet (G.).- Les bases légales du contrôle hygiénique du}

lait. Thèse Doct. vétérinaire, Alfort, 1940, 84 p. Vigot frères, éditeurs, 23, rue de l'Ecole-de-Médecine, Paris.

Cette thèse contient une documentation très intéressante pour tous ceux qui s'intéressent au contrôle hygiénique du lait, car elle présente un exposé des lois, décrets et règlements qui régissent cette importante question en France et à l'étranger. Ce travail témoigne d'une connaissance réelle du probleme et diffère des compilations que l'on rencontre si souvent.

L'introduction pose le problème : l'industrie laitière est capitale pour de nombreux pays, le lait est un aliment essentiel qui, "à lui seul, est la cause de plus de maladies et de morts que tous les autres aliments réunis ", le marché du lait doit être organisé du point de vue économique et les fraudes doivent être sévèrement punies, mais l'hygiène du lait doit être l'objet d'égales préoccupations.

Dans la première partie sont exposés les points essentiels de la réglementation française et les textes sont accompagnés de commentaires judicieux. 